\title{
FORECASTING THE MAIN STRUCTURAL CHANGES IN AGRICULTURE OF THE REPUBLIC OF SERBIA
}

\author{
Jelena Stanojević ${ }^{1}$, Vladimir Radivojević ${ }^{2}$, Tanja Stanišic ${ }^{3}$ \\ *Corresponding author E-mail: jelena.stanojevic@pr.ac.rs
}

\begin{abstract}
A R T I C LE IN F O
A B S T R A C T

Original Article

Received: 31 May 2021

Accepted: 30 August 2021

doi:10.5937/ekoPolj2103759S

UDC 338.341.4:63(497.11)

Keywords:

structural changes, agricultural output, forecast, Republic of Serbia

JEL: D2, O11, O13, Q10, Q13

Structural changes in agriculture, rural areas and regions, as well as in economic activities related to the production and trade of agricultural products, most often occur as a response to periodic changes in general economic and social conditions. In order to formulate effective policies and strategies, policy makers need adequate information about the main structural changes in agriculture. Therefore, based on the information framework of the Statistical Office of the Republic of Serbia, the article primarily focuses on changes of agricultural output in Serbia, respectively changes in crop and animal production, and agricultural services. The research aims to analyze changes in the structure of agricultural output in the period from 2007 to 2019 in order to identify its future trend by applying the forecast function. The research results show that the agricultural production of goods and services will keep the positive trend with a dominant share of crop production.
\end{abstract}

(C) 2021 EA. All rights reserved.

\section{Introduction}

Economic development of a country is mainly characterized by changes in the structure of economic activity. The varying development degree of economic sectors causes a change in their relative importance in the economy over time. Factors of these changes are complex and may include: changes in demand, introduction of new products and processes, different

1 Jelena Stanojević, PhD, Teaching Assistant with Doctorate, Faculty of Economics, University of Priština in Kosovska Mitrovica, Kolašinska 156, 38220 Kosovska Mitrovica, Serbia, Phone: +381642967067, E-mail: jelena.stanojevic@pr.ac.rs, ORCID ID (https:// orcid.org/0000-0002-1353-4349)

2 Vladimir Radivojević, PhD, Assistant Professor, Faculty of Economics, University of Priština in Kosovska Mitrovica, Kolašinska 156, 38220 Kosovska Mitrovica, Serbia, Phone: +381641468356, E-mail: vladimir.radivojevic@pr.ac.rs, ORCID ID (https://orcid. org/0000-0002-3928-0623)

3 Tanja Stanišić, PhD, Associate Professor, Faculty of Hotel Management and Tourism in Vrnjačka Banja, University of Kragujevac, Vojvodjanska bb, 36210 Vrnjačka Banja, Serbia, Phone: +381644941542, E-mail: tanja.stanisic@,kg.ac.rs, ORCID ID (https://orcid. org/0000-0001-5809-794X)

http://ea.bg.ac.rs 
possibilities among sectors in terms of technological progress and substitution of factors, changes in the governmental role in carrying out an economic activity, changes in the pattern of international competitiveness, etc. (Kenneth et al., 1992).

The process of economic development mostly implies the reallocation of resources from agriculture to non-agricultural activities. While most economists agree that a structural transformation stems from productivity growth, there is no consensus of whether technological progress is more important in agriculture or industry. Bearing in mind the impassive, absolute significance of the agricultural sector in the economy, it is important to understand its key structural changes (Johnston, 1990) and their effect on the overall economy.

Structural changes have occurred in agriculture of Serbia for the last decades. In Serbia agricultural sector traditionally plays a crucial role in the national economy, acting as a backbone of economic development. It has a significant economic and social function in the society. The social aspect has been expressed for years, putting agriculture in the function of preserving the living standards of the population and reducing poverty. On the other hand, the economic role of agriculture is multiple. Although agriculture for decades has been only considered as the production of agricultural products, nowadays processing, marketing and distribution of crop and animal products are considered as an integral part of agriculture (Đekić et al., 2013). Agricultural products are largely represented in the trade balance of the country, primarily on exports, contributing to the economic development with a still significant participation in the national product of Serbia (Božić et al, 2020, Stanojević \& Stanišić, 2015). In addition to providing food and raw materials for industry, agriculture in Serbia provides employment opportunities to rural population. This is especially important given that agriculture, in addition to providing livelihoods for farmers, contributes to alleviating high unemployment in rural areas, and in the entire economy (Halloran \& Archer, 2008).

The paper focuses on structural changes in the agricultural production of goods and services as one of the main structural changes in agriculture. After examining the theoretical framework of the concept of structural changes and the role of agriculture in the national economy, structural changes in Serbian agriculture are elaborated from the aspect of agricultural output. Further, based on the trend function, future trend in crop and animal production and agricultural services has been forecasted. The aim of this research is to identify changes in agricultural output by analyzing its branches, but also to forecast a further course of their movements in a five years period.

\section{Theoretical framework: The conceptual basis of structural changes in agriculture and its roles in the economy}

Structural changes in production, but also the employment of individual sectors at the expense of other sectors during the development and growth process, were recognized as a feature of modern economic growth by economists Forast (1949) and Simon Kuznets (1956). Based on historical data from industrialized countries, both authors have noted a 
development pattern reflected in a decline of the relative importance of agriculture, rapid industry growth and gradual increase in the importance of service sector in the economy (Raiser et al., 2003).

Early classical theory has described economic development as a process that leads to the systematic reallocation of production factors from the primary sector, characterized by low productivity, traditional technology and low income, to the modern industrial sector with high productivity rates and high income (Adelman, 1999). Agriculture has been considered as a traditional low productivity sector that passively contributes to development, providing food and employment. The significance of agriculture was expected to decline with a further development of economies (Kumar et al., 2019). Despite of this, agriculture is still considered necessary for development and economy transformation from traditional to modern (Konieczna \& Konieczny, 2018).

Two basic agricultural characteristics in the early stages of development prove its role and place in economic development. First, agriculture produces products that directly meet basic human needs. Secondly, agricultural production combines the human knowledge, skills and work with natural resources. Given that natural resources are available and free, theorists of economic development initially believed that agriculture can develop independently of other economic activities. However, practice has shown that agricultural dependence on scarce surface of the country limits its progress, disabling the value of agricultural production to proportionally follow the growth of labour supply and technology. This is one of the reasons for diminishing returns in agriculture (Xinshen et al., 2007). On the other hand, the tendency to meet basic human needs and avoid stagnation in development implies that agriculture should ultimately develop at the same rate as the population.

The agricultural sector still has a strategic role in the process of economic development of a country. This sector has played a significant role and contributed greatly to the economic prosperity of developed countries, and its role in developing countries is still vital (Ark, 1995). The history of developed countries has shown a crucial contribution of the agricultural sector to the process of industrialization and overall economic development indicating that development of agricultural and industrial sectors are not alternatives, but complementary in the same process (Downes \& Stoeckel, 2006). The fact that agriculture in most developing countries greatly contributes to the national product and still employs a large part of the workforce makes it unavoidable in discussions on the progress of the national economy (Gerdien \& Pim, 2007). As the gross domestic product per capita is lower, the focus of economic development is on the primary sector of the economy. Nevertheless, the role of agriculture in economic growth and development has changed dramatically in the last decades (Xiahui, 2020).

Certain characteristics of agriculture as a primary sector determine its specific role in the economy and indicate a difference from other sectors (Gardner \& Rausser, 2002). First, the primary sector is characterized by homogeneous products, which is one of the conditions for the absence of imperfect competition. One of reasons for greater flexibility in prices of agricultural product compared to the prices of industrial products is freely adjustment of 
agricultural prices to the conditions of (almost) perfect competition. Although the influence on the determination of agricultural prices is possible through the cartel of the commodity market, various policies and state interventions, etc., it is less evident than for industrial products and prices.

The second important characteristic of the primary sector is foreseen in the seasonal and climate changes impact on the agricultural production. This results in seasonal fluctuations in prices, where even the insurance of such cases is usually not very helpful. Prices of primary products are affected by unpredictable factors, difficult to prevent and control.

The third characteristic of agricultural activities refers to the fact that in most countries they are carried out by a large part of the population (primarily in low-income countries). For that reason, the government strives to regulate the agricultural sector through agricultural policy. That is inspired by not only social reasons, but also by the need to protect the environment and prevent its endangering.

The fourth characteristic of the primary sector is reflected in the production that mainly relies on a non-productive factor of production - a land which is physically limited and whose productivity cannot be indefinitely increased. Although a modern agricultural technology and innovation can significantly contribute to overcoming this weakness, the land scarcity is still a major problem in most cases and significantly affects agricultural prices and the market (Gardner \& Rausser, 2002).

Analyzing the role of agriculture in the national economy, the classics stated that the most developed countries have the so-called dual economy (Lewis, 1994) reflected in a lower labour productivity in agriculture compared to industry, which leads to a movement of workers from agriculture to non-agricultural sectors. It was considered that the nonagricultural innovation and technological changes are independent of agriculture, so agricultural workers and capital are needed in order to meet a demand for labour force and financial and capital investments in industry. This assumption supports the claim that agricultural and industrial revolutions always occur at the same time. Also, the economy in which agriculture stagnates, does not record the development of industry (Lewis, 1994). The claim of the classic that higher agricultural surpluses are necessary for financing industrial development are not relevant today, taking into account the liberalization of capital markets where investments in most countries still depend on domestic savings (Xinshen et al., 2007).

In addition to the role which agriculture has in securing labour and capital for industry, classics also emphasized its importance in food security leading to the sustainable economic development of the national economy. If traditional agriculture stagnates, employment growth in the non-agricultural sector would lead to food shortages. The increase in food prices would increase the cost of living, especially in lower income households that have a higher proportion of food costs in total costs. Pressure to increase salaries in the nonagricultural sector would threaten its growth, especially in the early stage of development when technology is dominantly labour intensive. This is known as the so-called Rickard's trap, which appears as a foundation of many economic theorists. Accordingly, successful 
industrialization is not possible without a parallel effort to increase food production in order to avoid a danger of falling into the so-called Ricard's trap (Hayami \& Godo, 2005).

Although economic development theorists observed the development of agriculture as an essential component and prerequisite for the development of the entire national economy, in the second half of the last century many countries tried to speed up the process of industrialization through over-taxation of agriculture. After that period prevailed the opinion that agriculture has not only a passive, but also an active role in economic development (Nikolić et al., 2010).

The transformation of traditional into modern agriculture has revealed its potential in terms of contribution to development. Scientific-based technology adapted to the ecological conditions of the country, becomes fundamental for the agricultural development. Advances in mechanical and biological technology helped to overcome shortcomings from the perspective of the land and workers (Langemejer \& Boehlje, 2017). Successful agricultural innovations represent a process that reflects natural resources, the level of supply and demand for agricultural inputs and products, as well as the motivation of farmers, scientists, and in general private and public sector. Therefore, the growth of agricultural productivity implies a link between agriculture and non-agricultural sector. In other words, as originally claimed by the theoreticians, the agricultural development does not take place independently of non-agricultural sector (Xinshen et al., 2007).

The role of agriculture in the national economy has been modified due to the changes that have occurred compared to other sectors in the economy, as well as within agriculture itself (Xinshen et al., 2007). The relative importance of agriculture for the economic growth of a country has been reduced over time. This is reflected in a decreased share of agriculture in the gross domestic product in relation to industry and services sector (Lee, 1992). Further, the participation of the agricultural population in economically developed countries has been reduced. The reason for the redirection of the agricultural population in non-agricultural activities is due to the intensification of agricultural production and its productivity growth (Afsar \& Hossain, 2020). On the other hand, the role of agriculture in providing food that meets essential needs of the population is irreplaceable, i.e. the absolute importance of agriculture remains unchanged. Moreover, its role in providing foodstuffs is expanding due to the need to produce increased amount of agricultural products under changing circumstances (population growth, increasingly scarce natural resources, changed climate, etc.). However, the agricultural production has undergone multiple changes in proportion between crop and animal production, as well as in a type of production that dominate (Gardner \& Rausser, 2002).

\section{Information base, research methods and research questions}

The research aim of the study is to conduct a comprehensive research and analysis of structural changes in agricultural output, respectively in crop and animal production and agricultural services in Serbia. 
Considering the aim of the research, this paper focuses on the following research questions:

a) Have structural changes in Serbian agriculture reflected reduction in the total value of agricultural output in the analyzed period?

b) Has the relative share of crop and animal production in total agricultural production of Serbia recorded any significant change in the analyzed period?

c) Based on the forecast of agricultural output, is there expected a further growth of crop and animal production, and agricultural services?

Research methods applied in the study are descriptive statistics and forecast trend. The forecast trend finds its application in determining the future movement of crop and animal production and agricultural services in Serbia. The Statistical Yearbook and Agricultural Economic Accounts of the Statistical Office of the Republic of Serbia in the period from 2007 to 2019 represent the information basis of this research.

\section{Research results and discussion}

\section{Structural changes in the agricultural output of the Republic of Serbia}

In the total value of agricultural production in Serbia traditionally dominates crop production due to the natural wealth and favorable climate for many different types of plants. The share of crop production is around two thirds of the total value, while animal production accounts for one-third. The share of agricultural services in total agricultural output, on the other hand, is only around 3 percentages.

The volume of agricultural production of goods and services of Serbia in the period from 2007 to 2019, as the analyzed period of this research, varies significantly, mainly due to adverse climate changes, but also given the other market fluctuations and influences. The absolute production values of agricultural goods and services in Serbia are expressed in producer prices of previous periods and represent the volume of production (Table 1).

The agricultural output has been increased almost twice in the observed period (from 330,174 mil. dinars in 2007 to 605,291 mil. dinars in 2019), even though the values fluctuated during this thirteen-year period. The most significant increase in agricultural output was recorded in 2008 compared to 2007 (27\%). In the following years, both positive and negative volume growth changes have alternated, ending up with the overall rise in the agricultural output in 2019. The maximum value of agricultural output in general, but also in agricultural goods output and agricultural services, was achieved in 2019. Such movements are a result of large oscillations in the value of crop production since it accounts for two thirds of the total value of agricultural production and thus mostly affect the overall result. Given that the crop production reached its highest value in 2019, it was expected to have this kind of results for agricultural output as well. On the other hand, animal production achieved the highest values in 2014 . 
The highest growth in crop production was recorded in 2008 (28\%) and 2010 (24\%), and the remaining years recorded lower growth rate, and even negative $(2009,2012,2015$, 2017). While both crop and animal production recorded the biggest volume growth in 2008, animal production reached its pick in 2014 and ended up in 2019 with the value higher for $70 \%$ then in 2007 . Agricultural services in the whole analyzed period from 2007 to 2019 record the value which is incomparably lower than value of agricultural goods output. Namely, the value of agricultural services is almost 40 times lower than the value of agricultural goods output in 2019. Even though the agricultural services record the highest value in 2019 (15,313 mil. dinars) which has been increased for $63 \%$ compared to 2007, the biggest increase in volume was recorded in $2008(11 \%)$ and $2009(12 \%)$, while negative volume changes occur in $2010(-5 \%), 2011(-2 \%), 2015$ (-8\%) and 2017 (-8\%). 
Table 1. Agricultural output at current producer prices in Serbia in the period 2007-2019, in mil. dinars

\begin{tabular}{|c|c|c|c|c|c|c|c|c|c|c|c|c|c|c|c|c|c|c|c|c|c|c|}
\hline 晜 & 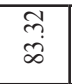 & 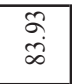 & $\begin{array}{l}\text { वे } \\
\text { ूे }\end{array}$ & 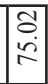 & $\mid \begin{array}{c}2 \\
0 \\
0 \\
0 \\
0\end{array}$ & 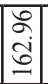 & $\overrightarrow{\vec{\rho}}$ & $\frac{\widehat{\partial}}{\vec{\gamma}}$ & \begin{tabular}{|l|} 
\\
\\
$\vdots$ \\
$\vdots$
\end{tabular} & 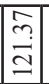 & 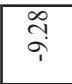 & $\begin{array}{l}n \\
\tilde{6} \\
0\end{array}$ & $\begin{array}{l}\stackrel{0}{2} \\
\stackrel{0}{\circ}\end{array}$ & 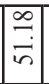 & $\begin{array}{l}\text { 品 } \\
\text { aे }\end{array}$ & $\mid \begin{array}{c}0 \\
0 \\
0 \\
0 \\
0\end{array}$ & 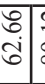 & $\begin{array}{c}\because \\
\dot{\infty}\end{array}$ & $=$ & 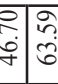 & 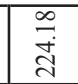 & $\begin{array}{l}\text { तु } \\
\text { రె }\end{array}$ \\
\hline ิㅡㄹ & $\begin{array}{l}\overline{\text { ते }} \\
\hat{\text { है }}\end{array}$ & 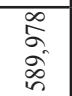 & $\begin{array}{l}\text { ते } \\
\text { əे } \\
\vec{\gamma}\end{array}$ & 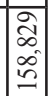 & $\begin{array}{l}\frac{n}{n} \\
6 \\
\end{array}$ & \begin{tabular}{|l|}
$\tilde{n}$ \\
$\tilde{n}$ \\
$\tilde{m}$
\end{tabular} & $\begin{array}{l}\frac{+}{n} \\
\stackrel{n}{m} \\
m\end{array}$ & $\begin{array}{l}2 \\
\infty \\
\infty \\
= \\
=\end{array}$ & \begin{tabular}{|l|} 
\\
\\
0 \\
6
\end{tabular} & 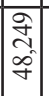 & $\tilde{n}$ & $\begin{array}{l}\text { 号 } \\
\text { 品 } \\
\text { = }\end{array}$ & $\begin{array}{l}\hat{\vdots} \\
\overrightarrow{\vec{a}}\end{array}$ & 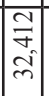 & 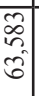 & ले & $\begin{array}{l}\tilde{I} \\
\stackrel{0}{0} \\
0\end{array}$ & $\begin{array}{l}\text { 管 } \\
\qquad\end{array}$ & 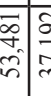 & & ণ্ & $\begin{array}{l}\stackrel{m}{m} \\
\stackrel{n}{n}\end{array}$ \\
\hline స్ & 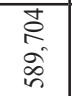 & 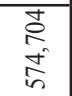 & $\begin{array}{l}\frac{J}{n} \\
\infty \\
\infty \\
\infty\end{array}$ & \begin{tabular}{|l|} 
\\
0 \\
0 \\
$n$ \\
$n$
\end{tabular} & $\mid \begin{array}{c}\vec{n} \\
\hat{n} \\
\hat{\sigma}\end{array}$ & 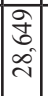 & $\begin{array}{l}\hat{0} \\
\text { iे }\end{array}$ & \begin{tabular}{|l|}
$\infty$ \\
$\stackrel{\infty}{n}$ \\
\end{tabular} \mid & $\mid$\begin{tabular}{|c|} 
\\
$\infty$ \\
0 \\
0 \\
0 \\
0
\end{tabular} & 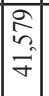 & గ్రి & $\begin{array}{l}\frac{8}{6} \\
\stackrel{6}{=}\end{array}$ & 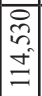 & $\mid$\begin{tabular}{c|}
$\hat{\infty}$ \\
0 \\
$m$ \\
$m$
\end{tabular} & 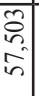 & i & $\begin{array}{c}\text { ते } \\
\infty \\
\infty\end{array}$ & 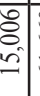 & \begin{tabular}{l|l}
8 \\
0 \\
6 \\
6 & 5
\end{tabular} & 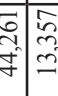 & $\begin{array}{l}\text { F } \\
\text { o } \\
\stackrel{+}{*}\end{array}$ & $\begin{array}{l}\overline{8} \\
\ddot{2}\end{array}$ \\
\hline స్ & 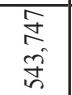 & 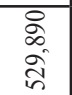 & 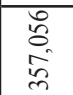 & $\begin{array}{l}8 \\
2 \\
2 \\
= \\
=\end{array}$ & 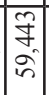 & 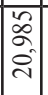 & $\begin{array}{l}\infty \\
\hat{n} \\
\hat{n} \\
\tilde{n}\end{array}$ & \begin{tabular}{|l}
$\hat{\infty}$ \\
$\stackrel{0}{=}$ \\
$=$
\end{tabular} & 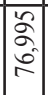 & 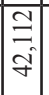 & $\aleph_{n}^{\infty}$ & $\begin{array}{l}\text { tu } \\
\text { o } \\
\text { i } \\
=\end{array}$ & 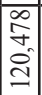 & 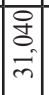 & $\begin{array}{l}2 \\
\frac{1}{8} \\
8\end{array}$ & \begin{tabular}{|l|}
$\infty$ \\
$\infty$ \\
$m$
\end{tabular} & $\begin{array}{l}0 \\
\vec{\sigma} \\
\infty \\
\infty\end{array}$ & $\begin{array}{ll} \\
\\
\end{array}$ & 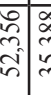 & & 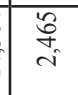 & 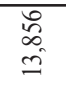 \\
\hline ํㅡㄹ & $\begin{array}{l}\infty \\
\infty \\
\infty \\
\infty \\
\infty\end{array}$ & $\begin{array}{l}\frac{\infty}{\infty} \\
+ \\
i \\
i\end{array}$ & $\begin{array}{l}\text { \&े } \\
\text { वे } \\
\text { Fे }\end{array}$ & 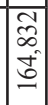 & 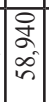 & $\begin{array}{l}\tilde{b} \\
0 \\
\hat{\sim} \\
\end{array}$ & $\begin{array}{l}\hat{2} \\
\hat{n} \\
\hat{q}\end{array}$ & 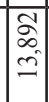 & $\begin{array}{l}\vec{\partial} \\
\vec{t}\end{array}$ & 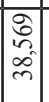 & $\tilde{n}$ & 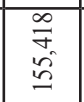 & 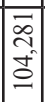 & \begin{tabular}{|c|}
$\hat{n}$ \\
$\hat{2}$ \\
$\hat{n}$ \\
$\tilde{e}$
\end{tabular} & $\begin{array}{l}\mathrm{N} \\
\mathrm{A} \\
\mathrm{n} \\
\mathrm{n}\end{array}$ & $\overrightarrow{0}$ & $\begin{array}{l}\infty \\
\text { ळे } \\
\text { nो }\end{array}$ & $\begin{array}{l}\text { ने } \\
\text { ले }\end{array}$ & \begin{tabular}{l|l}
$m$ & $\infty$ \\
$m$ & \\
$n$ &
\end{tabular} & & 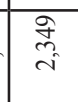 & $\begin{array}{l}8 \\
8 \\
0\end{array}$ \\
\hline ֻั) & 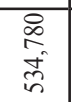 & 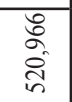 & $\begin{array}{l}\widehat{\widehat{a}} \\
\overrightarrow{\tilde{n}}\end{array}$ & \begin{tabular}{|l|} 
\\
2 \\
2 \\
2 \\
\\
\end{tabular} & 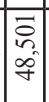 & \begin{tabular}{|l|}
$\tilde{n}$ \\
\\
$=$
\end{tabular} & $\begin{array}{l}\infty \\
\infty \\
i \\
n \\
n\end{array}$ & 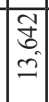 & 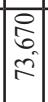 & 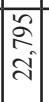 & $\tilde{n}$ & $\begin{array}{l}\infty \\
\tilde{\delta} \\
\hat{\sigma}\end{array}$ & 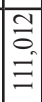 & \begin{tabular}{|l|}
$\hat{2}$ \\
$\bar{n}$ \\
$\bar{m}$
\end{tabular} & $\begin{array}{l}\infty \\
0 \\
\text { in } \\
\text { n. }\end{array}$ & $\nabla$ & $\begin{array}{l}\overrightarrow{5} \\
\infty\end{array}$ & $\frac{6}{m}$ & $\begin{array}{l}0 \\
\text { an } \\
\infty \\
n \\
n\end{array}$ & $\begin{array}{c}\frac{0}{m} \\
\text { ch- } \\
m\end{array}$ & 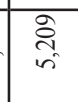 & $\begin{array}{l}\vec{J} \\
\infty \\
\stackrel{\sim}{\eta}\end{array}$ \\
\hline$\stackrel{ \pm}{\stackrel{\Xi}{\sim}}$ & $\begin{array}{l}\stackrel{8}{ } \\
\text { m } \\
\stackrel{\infty}{\infty} \\
\stackrel{n}{n}\end{array}$ & $\begin{array}{l}\stackrel{0}{N} \\
o \\
\stackrel{0}{n} \\
\stackrel{n}{n}\end{array}$ & $\begin{array}{l}\frac{\infty}{+\infty} \\
\stackrel{0}{0} \\
\stackrel{-}{0}\end{array}$ & 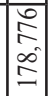 & $\begin{array}{l}\tilde{2} \\
\tilde{n} \\
\dot{n} \\
\end{array}$ & \begin{tabular}{|c|}
$\infty$ \\
0 \\
0 \\
$\approx$ \\
0
\end{tabular} & $\begin{array}{l}m \\
0 \\
0 \\
\stackrel{N}{N}\end{array}$ & 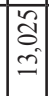 & $\mid \begin{array}{l}2 \\
\infty \\
\infty \\
0 \\
0 \\
n\end{array}$ & \begin{tabular}{|l|}
$\overrightarrow{\widehat{G}}$ \\
$\overrightarrow{\tilde{m}}$
\end{tabular} & 命 & $\begin{array}{l}0 \\
\widetilde{1} \\
\infty \\
\stackrel{0}{=}\end{array}$ & 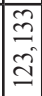 & 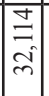 & $\begin{array}{l}3 \\
\vdots \\
\hat{3} \\
0\end{array}$ & $\bar{n}$ & $\begin{array}{l}\infty \\
0 \\
0 \\
0\end{array}$ & 命 & 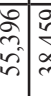 & 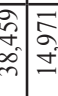 & $\stackrel{8}{\circ}$ & $\begin{array}{l}\text { d } \\
\text { in }\end{array}$ \\
\hline ڤิ & $\begin{array}{l}\overrightarrow{\tilde{n}} \\
\hat{n} \\
\stackrel{n}{n}\end{array}$ & $\begin{array}{l}\text { ô } \\
\text { î } \\
\hat{n}\end{array}$ & $\begin{array}{l}\tilde{D}^{-} \\
\infty \\
\infty \\
\infty\end{array}$ & 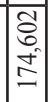 & 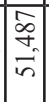 & 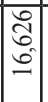 & $\begin{array}{l}\stackrel{n}{n} \\
\stackrel{n}{n} \\
\end{array}$ & $\begin{array}{l}2 \\
0 \\
2 \\
2\end{array}$ & 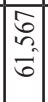 & $\begin{array}{l}n \\
\tilde{n} \\
\tilde{n}\end{array}$ & 是 & $\begin{array}{l}\stackrel{+}{d} \\
\stackrel{\text { d }}{=}\end{array}$ & $\mid \begin{array}{l}\tilde{\partial} \\
\infty \\
\infty \\
= \\
=\end{array}$ & $\left|\begin{array}{c}\mathbf{y} \\
\mathfrak{9} \\
\text { in }\end{array}\right|$ & $\begin{array}{l}0 \\
0 \\
\vdots \\
\vdots \\
0\end{array}$ & ڤે̊ & $\frac{\vec{a}}{\infty}$ & $\begin{array}{l}2 \\
\overrightarrow{5} \\
=\end{array}$ & $\begin{array}{l}\hat{n} \\
\text { 2. } \\
\text { n. }\end{array}$ & $\begin{array}{c}\infty \\
0 \\
\infty \\
\infty \\
\infty\end{array}$ & $\begin{array}{l}\text { Pे } \\
\text { a } \\
\text { in }\end{array}$ & $\begin{array}{l}\mathcal{y} \\
g\end{array}$ \\
\hline స్ㄱ & 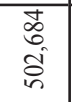 & $\begin{array}{l}\hat{\sigma} \\
\hat{\sigma} \\
\bar{\sigma}\end{array}$ & $\begin{array}{l}\overrightarrow{\vec{f}} \\
\stackrel{+}{d} \\
\text { nd }\end{array}$ & 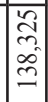 & 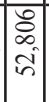 & \begin{tabular}{|l|} 
\\
$\hat{\sigma}$ \\
0 \\
0 \\
\end{tabular} & 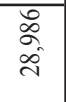 & 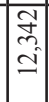 & 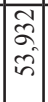 & \begin{tabular}{|c|} 
\\
$\widehat{a}$ \\
$\infty$ \\
$-\infty$
\end{tabular} & f & \begin{tabular}{l}
$\stackrel{0}{+}$ \\
\multirow{6}{0}{}
\end{tabular} & 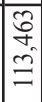 & \begin{tabular}{|c|}
$\hat{m}$ \\
$\hat{m}$ \\
$\hat{m}$
\end{tabular} & 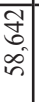 & ह̂. & $\begin{array}{l}\vec{a} \\
\infty \\
- \\
-\end{array}$ & 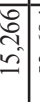 & 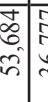 & 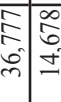 & $\begin{array}{l}\text { ते } \\
\text { ì }\end{array}$ & $\begin{array}{l}\hat{\infty} \\
\stackrel{=}{=}\end{array}$ \\
\hline$\overline{\bar{\sim}}$ & 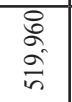 & $\begin{array}{l}\stackrel{\text { an }}{a} \\
\text { oे }\end{array}$ & $\begin{array}{l}\hat{\sigma} \\
\hat{O} \\
\text { مे }\end{array}$ & 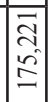 & \begin{tabular}{|l|}
2 \\
6 \\
6 \\
0 \\
+ \\
+
\end{tabular} & 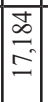 & $\begin{array}{l}\stackrel{0}{d} \\
\underset{\sim}{N}\end{array}$ & \begin{tabular}{|l|}
2 \\
$\infty$ \\
$\infty$ \\
$=$ \\
\end{tabular} & \begin{tabular}{|c|} 
\\
$\infty$ \\
$\infty$ \\
0 \\
0 \\
$n$
\end{tabular} & \begin{tabular}{|c|}
\multirow{2}{*}{} \\
ते
\end{tabular} & $\stackrel{n}{n}$ & 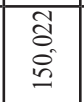 & 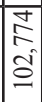 & 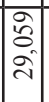 & 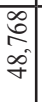 & 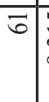 & $\begin{array}{l}\frac{n}{n} \\
2 \\
a\end{array}$ & $\begin{array}{l}1 \\
\vdots \\
n \\
n \\
n\end{array}$ & 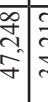 & \begin{tabular}{l|l}
$\mathrm{T}$ & 0 \\
$\mathrm{n}$ & $\infty$ \\
$\tilde{m}$ & 0
\end{tabular} & $\begin{array}{l}\text { त̃ } \\
\text { î }\end{array}$ & $\begin{array}{l}\text { 志 } \\
\infty \\
0 \\
0\end{array}$ \\
\hline 을 & $\begin{array}{l}\bar{\Xi} \\
\vec{\infty} \\
\dot{0} \\
\dot{0}\end{array}$ & 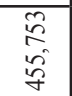 & $\begin{array}{l}\vec{\infty} \\
\infty \\
\infty \\
\infty\end{array}$ & 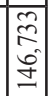 & $\left|\begin{array}{l}\hat{\sigma} \\
\overrightarrow{0} \\
\dot{f}\end{array}\right|$ & \begin{tabular}{|l|}
$\overrightarrow{\mathbf{b}}$ \\
$\stackrel{-}{=}$
\end{tabular} & 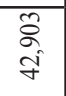 & \begin{tabular}{|l|} 
\\
0 \\
0 \\
$=$
\end{tabular} & $\begin{array}{l}\hat{2} \\
\vec{\gamma} \\
\vec{\gamma}\end{array}$ & \begin{tabular}{|l|} 
\\
$\infty$ \\
$\infty$ \\
$=$ \\
\end{tabular} & बे & 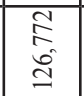 & 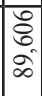 & 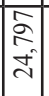 & 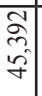 & 5 & $\begin{array}{l}0 \\
\text { n. } \\
\infty\end{array}$ & $\begin{array}{c}\text { 命 } \\
0 \\
0 \\
0\end{array}$ & 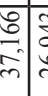 & 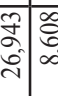 & $\stackrel{n}{\sigma}$ & $\begin{array}{l}\infty_{0}^{\infty} \\
\stackrel{0}{0} \\
=\end{array}$ \\
\hline ఫ్సి & $\begin{array}{l}\overrightarrow{\vec{n}} \\
\infty \\
\tilde{\sigma} \\
\dot{q}\end{array}$ & $\begin{array}{l}\overrightarrow{\mathrm{N}} \\
\text { ळू } \\
\text { ळे }\end{array}$ & $\begin{array}{l}\overrightarrow{0} \\
\hat{\tilde{b}} \\
\text { v }\end{array}$ & \begin{tabular}{|l|}
+ \\
$\infty$ \\
2 \\
0 \\
$\varrho$ \\
$=$
\end{tabular} & $\mid$\begin{tabular}{c|}
$\tilde{n}$ \\
$\hat{\tilde{n}}$ \\
$\tilde{n}$
\end{tabular} & $\begin{array}{l}\infty \\
\infty \\
n \\
\pm \\
\end{array}$ & $\begin{array}{l}\hat{n} \\
\infty \\
\infty \\
\sim\end{array}$ & $\frac{a}{a}$ & \begin{tabular}{|c|} 
\\
\multirow{0}{0}{} \\
m \\
\end{tabular} & $\begin{array}{c}0 \\
m \\
m \\
m \\
m\end{array}$ & $\begin{array}{l}\infty \\
\tilde{n}\end{array}$ & $\begin{array}{l}\stackrel{a}{=} \\
\overrightarrow{=}\end{array}$ & $\begin{array}{l}n \\
\hat{\infty} \\
\hat{\alpha} \\
\alpha\end{array}$ & 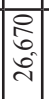 & $\begin{array}{l}\Omega \\
\vec{n} \\
\vec{n}\end{array}$ & 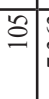 & $\begin{array}{l}\text { तु } \\
\text { ñ }\end{array}$ & $\begin{array}{l}3 \\
\\
0 \\
0 \\
0\end{array}$ & 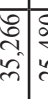 & \begin{tabular}{l|l}
8 & 9 \\
$\infty$ & 8 \\
+ & 0 \\
& $\infty$
\end{tabular} & $\stackrel{\tilde{m}}{=}$ & $\begin{array}{l}\stackrel{0}{6} \\
= \\
=\end{array}$ \\
\hline ఫ్ & $\begin{array}{l}\tilde{N} \\
\infty \\
\tilde{\sigma} \\
\vec{\sigma}\end{array}$ & 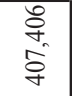 & 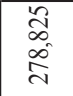 & \begin{tabular}{|l|}
$n$ \\
$\tilde{n}$ \\
$\tilde{n}$ \\
$n$
\end{tabular} & 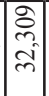 & 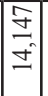 & $\begin{array}{l}\text { बे } \\
\text { o } \\
\text { d }\end{array}$ & \begin{tabular}{|l|} 
\\
$m$ \\
$\infty$ \\
$\infty$
\end{tabular} & 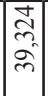 & 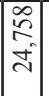 & $\vec{\sim}$ & $\begin{array}{l}\bar{\infty} \\
\text { n. } \\
\stackrel{0}{\simeq} \\
\simeq\end{array}$ & $\begin{array}{l}\frac{2}{2} \\
\frac{\infty}{\infty}\end{array}$ & \begin{tabular}{|l|} 
\\
$\stackrel{0}{\sim}$ \\
$\dot{d}$
\end{tabular} & 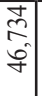 & $\stackrel{\infty}{=}$ & 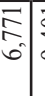 & 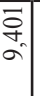 & 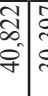 & 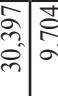 & $\overrightarrow{\mathbb{N}}$ & $\begin{array}{l}\stackrel{\mathscr{Y}}{\mathscr{y}} \\
\stackrel{0}{0}\end{array}$ \\
\hline ్ָ & 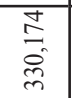 & $\begin{array}{l}\stackrel{\circ}{n} \\
\stackrel{n}{n} \\
m\end{array}$ & 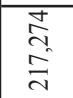 & 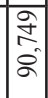 & 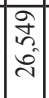 & \begin{tabular}{|l|}
$\overrightarrow{0}$ \\
$\hat{i}$
\end{tabular} & 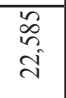 & \begin{tabular}{|l|}
$\infty$ \\
$\infty$ \\
$\infty$ \\
$\infty$
\end{tabular} & 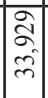 & \begin{tabular}{|l|} 
\\
2 \\
$-i$ \\
-1
\end{tabular} & $\bar{\infty}$ & 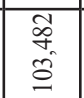 & $\begin{array}{l}\overline{8} \\
\text { बें }\end{array}$ & $\mid \begin{array}{l}\overrightarrow{\tilde{f}} \\
\vec{\sim} \\
\vec{v}\end{array}$ & $\begin{array}{l}\tilde{2} \\
\hat{n} \\
\tilde{m}\end{array}$ & Iิ & 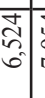 & 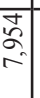 & 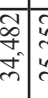 & 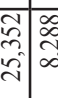 & F & $\begin{array}{l}\infty \\
\underset{\sigma}{\neq}\end{array}$ \\
\hline & 嵒 & 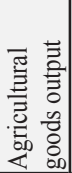 & 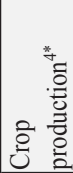 & 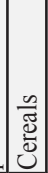 & 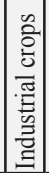 & \begin{tabular}{|c|} 
\\
0 \\
0 \\
0 \\
0 \\
0 \\
0 \\
0 \\
0 \\
0 \\
0 \\
0
\end{tabular} & 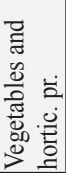 & 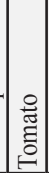 & & 竧 & 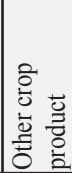 & 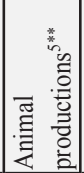 & 虽 & 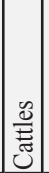 & 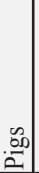 & 离 & "ెี & 量 & & & 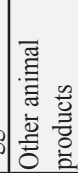 & 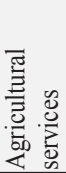 \\
\hline
\end{tabular}

Source: Statistical Office of the Republic of Serbia (c). (2009-2020). The Statistical Yearbook ${ }^{4}$ *Index of agricultural output change in 2019 compared to 2007

$4 *$ The value of crop production includes the production of cereals, industrial plants, fodder plants, vegetables and products of horticulture, fruit and other non-mentioned agricultural goods (Statistical Office of the Republic of Serbia (b), 2019)

$5 * *$ The value of animal production includes the production/breeding of livestock, poultry and other animals and livestock goods. The production of livestock goods includes the production of milk, eggs and other non-mentioned agricultural goods (Statistical Office of the Republic of Serbia (b), 2019) 
Table 2 shows the share of agricultural goods output and agricultural services in the total agricultural output in Serbia in the period 2007-2019, as well as a share of crop and animal production in agricultural output, including their branches.

Table 2. Agricultural output in Serbia for the period 2007-2019, in percentages

\begin{tabular}{|c|c|c|c|c|c|c|c|c|c|c|c|c|c|}
\hline & હิণ & 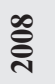 & ڤ્ণે & 을 & $\overline{\bar{ন}}$ & 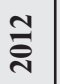 & 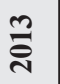 & $\underset{\bar{N}}{\stackrel{\Xi}{I}}$ & $\stackrel{n}{\frac{\pi}{\pi}}$ & 룰 & 귝 & $\stackrel{\infty}{\stackrel{\pi}{\pi}}$ & 를 \\
\hline Agricultural goods output & 97 & 98 & 97 & 98 & 98 & 98 & 98 & 97 & 97 & 97 & 97 & 97 & 97 \\
\hline Crop production & 66 & 67 & 65 & 70 & 69 & 65 & 67 & 67 & 66 & 71 & 66 & 68 & 68 \\
\hline Cereals & 27 & 32 & 27 & 31 & 34 & 28 & 31 & 31 & 26 & 28 & 21 & 27 & 26 \\
\hline Industrial crops & 8 & 8 & 8 & 10 & 9 & 11 & 9 & 9 & 9 & 10 & 11 & 11 & 10 \\
\hline Forage plants & 4 & 3 & 4 & 4 & 3 & 4 & 3 & 4 & 3 & 5 & 4 & 5 & 6 \\
\hline Vegetables and hort. prod. & 7 & 6 & 7 & 9 & 5 & 6 & 5 & 5 & 7 & 7 & 6 & 4 & 5 \\
\hline Tomato & 37 & 33 & 34 & 41 & 66 & 43 & 70 & 45 & 38 & 34 & 36 & 51 & 37 \\
\hline Fruits & 10 & 9 & 9 & 9 & 10 & 11 & 11 & 10 & 14 & 13 & 14 & 12 & 11 \\
\hline Wine & 7 & 6 & 8 & 4 & 5 & 4 & 5 & 6 & 4 & 7 & 8 & 7 & 8 \\
\hline Other crop product & 0 & 0 & 0 & 0 & 0 & 0 & 0 & 0 & 0 & 0 & 0 & 0 & 0 \\
\hline Animal productions & 31 & 31 & 32 & 27 & 29 & 33 & 31 & 31 & 32 & 26 & 32 & 30 & 29 \\
\hline Animals & 21 & 21 & 24 & 19 & 20 & 23 & 21 & 21 & 21 & 18 & 22 & 19 & 20 \\
\hline Cattles & 6 & 6 & 7 & 5 & 6 & 6 & 6 & 5 & 6 & 5 & 6 & 6 & 5 \\
\hline Pigs & 10 & 11 & 13 & 10 & 9 & 12 & 11 & 11 & 11 & 9 & 12 & 10 & 11 \\
\hline Equines & 0 & 0 & 0 & 0 & 0 & 0 & 0 & 0 & 0 & 0 & 0 & 0 & 0 \\
\hline Sheep and goats & 2 & 2 & 2 & 2 & 2 & 2 & 1 & 2 & 2 & 1 & 2 & 1 & 2 \\
\hline Poultry & 2 & 2 & 3 & 2 & 3 & 3 & 3 & 3 & 2 & 2 & 3 & 3 & 2 \\
\hline Other animals & 10 & 10 & 9 & 8 & 9 & 11 & 10 & 9 & 11 & 9 & 10 & 10 & 9 \\
\hline Milk & 8 & 7 & 6 & 6 & 7 & 7 & 7 & 7 & 7 & 6 & 7 & 8 & 6 \\
\hline Eggs & 3 & 2 & 2 & 2 & 2 & 3 & 2 & 3 & 3 & 2 & 3 & 2 & 2 \\
\hline Other animal products & 0 & 0 & 0 & 0 & 0 & 0 & 1 & 0 & 1 & 0 & 0 & 1 & 0 \\
\hline Agricultural services & 3 & 2 & 3 & 2 & 2 & 2 & 2 & 3 & 3 & 3 & 3 & 3 & 3 \\
\hline
\end{tabular}

Source: Authors' calculation

In the structure of agricultural output of Serbia in the period 2007-2019 dominates agricultural production of goods with around 97-98\% while agricultural services are included with only $2-3 \%$ in the whole analyzed period. Looking into the value of crop and animal production and their share in the total value of agricultural output, a dominant share has a crop production (around 67\%), while animal production accounts on average of only $30 \%$.

Within the crop production, the highest participation is mostly presented by tomato (around $40 \%$ ), cereals (around 28\%) and fruit (around 11\%). The share below 10\% within crop production is noticeable in industrial crops $(9 \%)$, forage plants $(4 \%)$, vegetables and products of horticulture $(6 \%)$, wine $(6 \%)$ and other crop products $(0.1 \%)$.

In the value structure of animal production, the dominant share in agricultural output records animals $(21 \%)$, pigs $(10 \%)$ and other animals $(10 \%)$, while all others under the animal production participate with less than $10 \%$ in agricultural output. 
Agricultural production in Serbia is mainly intended for sale on the market, usually from agricultural holdings that includes sale to other agricultural holdings, entities outside agriculture and exports. On average for the observed period, sale from agricultural holdings accounted for $80 \%$ of total agricultural production. The consumption of agricultural goods within the units, and for the needs of various agricultural activities (for example, the use of crop products for animal nutrition purposes) ranged between 8.4\% and 14.9\% (Statistical Office of the Republic of Serbia (b), 2019).

Only a small part of the production of agricultural holdings is intended for own consumption, which in the observed period 2007-2019 was $6 \%$ on average. However, in some years this participation was lower. Thus, in 2018 amounted to $4.7 \%$ and in 2019 was $4.8 \%$ of the total value of agricultural production (Statistical Office of the Republic of Serbia (b), 2019).

\section{Forecasting the movement of agricultural output in the Republic of Serbia}

Estimating the future trends of key agricultural indicators is considered as a useful tool for stakeholders (farmers, agricultural enterprises, state institutions, etc.). Given the crucial role that food production has in providing a social security of citizens, the state has a special interest in predicting as accurately as possible further changes in agriculture.

The state usually performs forecasts of agriculture, but on the other hand also appears as the main user of the results. Although the forecasting cannot provide an exact information about the future, but only prediction and probabilities about the further trend in some indicators based on the historical data, it often supports the decision-making process. When drafting different forecasts and apply various methods, it is of a high importance to take into account indicators that will ensure easier implementation of policies, providing technical and market assistance to the agricultural sector (Sanders, 2000).

Figures 1-3 show the forecasted values of crop production, animal production and agricultural services in Serbia for the next five years. Exponential smoothing forecast method has been applied based on the historical values from the period 2007-2019 available in the publications of the Statistical Office of the Republic of Serbia. A confidence interval of $95 \%$ indicates the probability of $95 \%$ for future values to occur.

The future value $(\mathrm{y})$ has been calculated based on the following formula for linear regression equation (Sanders, 2000):

$y=a+b x$

where:

the $a$ constant (intercept) is:

$a=\bar{y}-b \bar{x}$

the $b$ coefficient (slope of the line) is:

$b=\frac{\sum(x-\bar{x})(y-\bar{y})}{\sum(x-\bar{x})^{2}}$ 
Figure 1. Forecast tendencies of values of crop production in Serbia

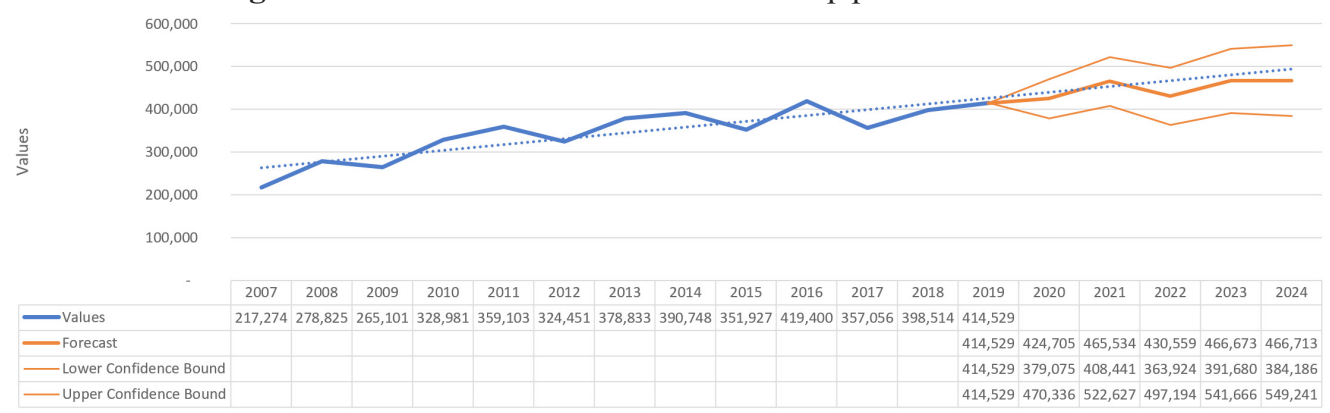

Source: Statistical Office of the Republic of Serbia \& authors' calculations

Figure 2. Forecast tendencies of values of animal production in Serbia

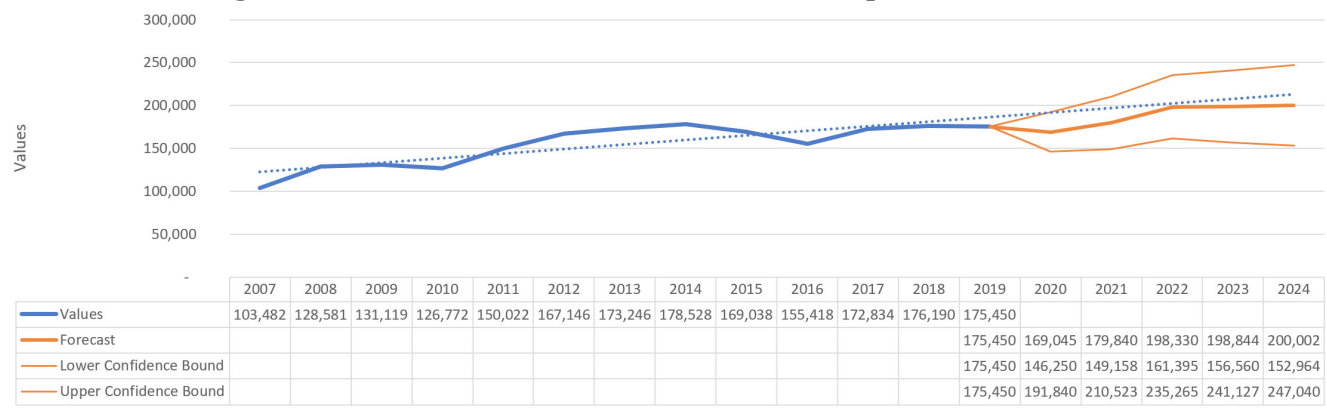

Source: Statistical Office of the Republic of Serbia \& authors' calculations

Figure 3. Forecast tendencies of values of agricultural services in Serbia

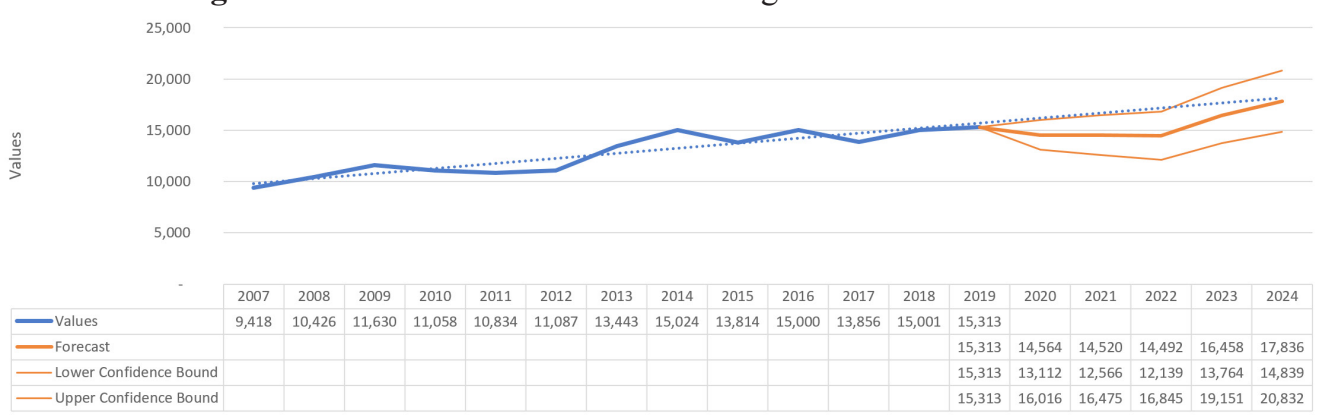

Source: Statistical Office of the Republic of Serbia \& authors' calculations

The figures 1, 2 and 3 show both historical, respectively realized values of crop and animal production and agricultural services, as well as the forecasted values for the next five years based on the historical data from 2007 to 2019. The forecasted values are ranged between the lower and upper limits of reliability, providing information about the interval for the future values to appear. Along with the historical and forecasted values, in all three figures are provided trends aiming to ensure easier observation of movements. Based on the forecast shown in Figures 1-3, crop production, animal production and agricultural services in Serbia have a tendency of further growth in the next five years. 


\section{Conclusion}

Agriculture in Serbia faces many challenges that caused different changes in its structure. The key challenge faced by agricultural and economic policy makers of Serbia is how to ensure a sustainable agricultural development process that will respond to the challenges of developed modern technology and increased demand in the market. Therefore, it is extremely important to ensure productivity growth, strengthen the agricultural market, stimulate investment, invest in research and development, improve links between agriculture and non-agricultural sector in rural areas, invest in human resources, encourage key branches of crop and animal production, and ensure regional cooperation of stakeholders. Although small and medium-sized agricultural holdings constitute almost the total number of agricultural holdings in Serbia, agriculture in the future should rely on large agricultural holdings specialized in certain agricultural production. This trend can be partly attributed to technical innovations, economies of scale, increased consolidation in food processing, and distribution and sales.

In addition to meeting the need for quality, diversified and food in sufficient quantities, agriculture is expected to contribute to overall economic development and poverty reduction, to face increased competition for alternative uses of scarce land and water resources, to adapt to climate changes and contributes to the conservation of biodiversity and the restoration of sensitive ecosystems, etc. However, one of the challenges that agriculture will be exposed in the coming period is the sustainable production of food. Climate changes will bring higher average temperatures, changes in precipitation, more frequent extreme phenomena, numerous threats to sustainable food security. In order to meet these challenges, a coordinated action of the private and public sector and civil society is needed, which will have to be adapted to specific circumstances.

Given the research results of this study reflected in the forecasted further positive trend in crop and animal production and agricultural services, it is expected that agriculture can meet the future needs, even though there is a room for potential improvement in the field of more intensive development of animal production, diverse crop production and various agricultural services.

\section{Conflict of interests}

The authors declare no conflict of interest.

\section{References}

1. Adelman, I. (1999). Fallacies in Development Theory and Their Implications for Policy. In M. \&. Stiglitz, Frontiers of development economics: The future in perspective (pp. 103-134). New York: World Bank.

2. Afsar, R. \& Hossain, M. (2020). Migration and Occupational Changes: Dreams and Realities of Better Livelihoods. Dhaka's Changing Landscape: Prospects for Economic Development, Social Change, and Shared Prosperity, 2020, ill. 
3. Ark, V. (1995). Sectoral Growth Accounting and Structural Change in Postwar Europe. Groningen Growth and Development Centre, University of Gronngen.

4. Božić Miljković, I., Kaličanin, K. \& Mitić, V. (2020). Foreign trade of agricultural products between Serbia and the European Union - problems and perspectives. Economic Outlook, 22(1), pp. 29-46.

5. Downes, P. \& Stoeckel, A. (2006). Drivers of Structural Change in the Australian Economy. Sydney: Centre for International Economics - Canberra \& Sydney.

6. Gardner, B.L. \& Rausser, G.C. (2002). Handbook of Agricultural Economics (T.2). Amsterdam: Elsevier.

7. Gerdien, M. \& Pim, R. (2007). The role of agriculture in development. Markets, Chains and Sustainable (T. 5). Wageningen: Wageningen University \& Research.

8. Halloran, J.M. \& Archer, D.W. (2008). External Economic Drivers and US Agricultural Production Systems. Renewable Agriculture and Food Systems, 23 (4), 296-303.

9. Hayami, Y. \& Godo, Y. (2005). Development Economics: From the Poverty to the Wealth of Nations. Oxford: Oxford University Press.

10. Johnston, W. (1990). Structural Change and the Recognition of Diversity. American Journal of Agricultural Economics.72, pp. 1109-1123. American Agricultural Economics Association.

11. Kenneth, D., Caroline, J.\& Lynk, E.L. (1992). Industrial Organization: Competition, Growth and Structural Change. London: Routledge.

12. Konieczna, J. \& Konieczny, D. (2018). Structural changes in polish agriculture and rural areas following the accession to the EU. International Multidisciplinary Scientific GeoConference Surveying Geology and Mining Ecology Management, SGEM, 18(2.2):971-978

13. Kumar, S., Singh, R. \& Sharma, A. (2019). Structural transformation in agriculture and economic growth: a study in Himachal Pradesh. Agricultural Situation in India. 76(9):18-29

14. Langemejer, M. \& Boehlje, M. (2017). Drivers of Consolidation and Structural Change in Production Agriculture. Economic Review, special issue, pp. 5-23.

15. Lee, M. (1992). A Survey of Agricultural Economics Literature: Agriculture in Economic Development 1940s to 1990s. Minneapolis: University of Minnesota Press.

16. Lewis, A. (1994). Economic Development with Unlimited Supplies of Labour. Y R. Kanth, Paradigs in Economic Development: classic perspectives, critiques, and reflections (pp. 59-98). Armonk: Routledge.

17. Raiser, M. Schaffer, M., Schuchhardt, J. (2003). Benchmarking structural change in transition. European Bank for Reconstruction and Development. 
18. Xiahui, L. (2020). Structural changes and economic growth in China over the past 40 years of reform and opening-up. China Political Economy, 2020, Vol. 3, Issue 1, pp. 19-38.

19. Xinshen, D., Hazell, P., Resnick, D.\& Thurlow, J. (2007). The Role of Agriculture in Development: Implications for Sub-Saharan Africa. Washington: International Food Policy Research Institute.

20. Đekić, S., Jovanović, S., Stanojević, J. (2013). The role of agriculture in ensuring food security. Dependability and Quality Management - ICDQM (pp. 639-644). Prijevor: Research Center for Dependability and Quality Management.

21. Nikolić, I., Filipović, S., \& Miljković, M. (2010). The potential of Serbian agribusiness sector. Industrija, 38(4), 203-222.

22. Sanders N.R. (2000). Forecasting guidelines and methods. In: Swamidass P.M. (eds) Encyclopedia of Production and Manufacturing Management. Springer, Boston, MA. https://doi.org/10.1007/1-4020-0612-8_362

23. Statistical Office of the Republic of Serbia (a). (2020). Retrieved: www.stat.gov.rs.

24. Statistical Office of the Republic of Serbia (b). (2019). Economic accounts for agriculture in the Republic of Serbia, 2009-2019. Belgrade.

25. Statistical Office of the Republic of Serbia (c). (2009-2020). The Statistical Yearbook.

26. Stanojević, J., Stanišić, T. (2015). Značaj agrarnog sektora kroz istoriju ekonomske misli. Peščanik, No 13, Istrorijski arhiv Nis. [in English: Stanojević, J., Stanišić, T. (2015). The importance of the agricultural sector throughout the history of economic thought. Peščanik, No 13, Historical Archives of Nis.] 